\title{
Overview of Phobos/Deimos Regolith Ion Sample Mission (PRISM) concept
}

Pamela Clark, Michael Collier, Micah Schaible, William M. Farrell, David Folta, et al.

Pamela Clark, Michael Collier, Micah Schaible, William M. Farrell, David Folta, Kyle M. Hughes, John W. Keller, Ben Malphrus, Andrew S. Rivkin, Scott Murchie, Dana Hurley, Jasper Halekas, Richard Vondrak, Timothy Stubbs, Rosemary Killen, Menelaos Sarantos, Sarah L. Jones, Jared Espley, Gina Dibraccio, "Overview of Phobos/Deimos Regolith lon Sample Mission (PRISM) concept," Proc. SPIE 10769, CubeSats and NanoSats for Remote Sensing II, 107690I (18 September 2018); doi: 10.1117/12.2322415

SPIE Event: SPIE Optical Engineering + Applications, 2018, San Diego, California, United States 


\title{
Overview of Phobos/Deimos Regolith Ion Sample Mission (PRISM) Concept
}

\author{
Pamela Clark*1,2, Michael Collier*2,3, Micah Schaible ${ }^{4}$, William M. Farrell ${ }^{2,3}$, David Folta ${ }^{3}$, Kyle M. \\ Hughes $^{3}$, John W. Keller ${ }^{2,3}$, Ben Malphrus ${ }^{5}$, Andrew S. Rivkin 6 , Scott Murchie ${ }^{6}$, Dana Hurley ${ }^{2,6}$, \\ Jasper Halekas ${ }^{2,7}$, Richard Vondrak ${ }^{2,3}$, Timothy Stubbs ${ }^{2,3}$, Rosemary Killen²,3 Menelaos Sarantos ${ }^{2,3}$, \\ Sarah L. Jones ${ }^{3}$, Jared Espley ${ }^{3}$, Gina Dibraccio ${ }^{3}$ \\ 1. Jet Propulsion Laboratory, California Institute of Technology, Pasadena, CA, 2. SSERVI/DREAM2, Ames Research \\ Center, Mountain View, CA, 3. NASA/GSFC, Greenbelt, MD, 4. Georgia Institute of Technology, Atlanta, GA, 5. \\ Morehead State University, Morehead, KY, 6. JHU/APL, Laurel, MD, 7. University of Iowa, Iowa City, IA \\ *Pamela.E.Clark@jpl.nasa.gov; phone 1818 393-3262; fax 1818 354-8887; jpl.nasa.gov
}

\begin{abstract}
Far more definitive information on composition is required to resolve the question of origin for the Martian moons Phobos and Deimos. Current infrared spectra of the objects are inconclusive due to the lack of strong diagnostic features. Definitive compositional measurements of Phobos could be obtained using in-situ X-ray, gamma-ray, or neutron spectroscopy or collecting and returning samples to Earth for analysis. We have proposed, in lieu of those methods, to derive Phobos and Deimos compositional data from secondary ion mass spectrometry (SIMS) measurements by calibrating the instrument to elemental abundance measurements made for known samples in the laboratory. We describe the Phobos/Deimos Regolith Ion Sample Mission (PRISM) concept here. PRISM utilizes a high-resolution TOF plasma composition analyzer to make SIMS measurements by observing the sputtered species from various locations of the moons' surfaces. In general, the SIMS technique and ion mass spectrometers complement and expand quadrupole mass spectrometer measurements by collecting ions that have been energized to higher energies, $50-100 \mathrm{eV}$, and making measurements at very low densities and pressures. Furthermore, because the TOF technique accepts all masses all the time, it obtains continuous measurements and does not require stepping through masses. The instrument would draw less than $10 \mathrm{~W}$ and weigh less than $5 \mathrm{~kg}$. The spacecraft, nominally a radiation-hardened $12 \mathrm{U}$ CubeSat, would use a low-thrust Solar Electric Propulsion system to send it on a two-year journey to Mars, where it would co-orbit with Deimos and then Phobos at distances as low as $27 \mathrm{~km}$.
\end{abstract}

Keywords: cubesat, 12U, SIMS, Phobos, Deimos, composition, origin

\section{SOLAR SYSTEM SCIENCE CONTEXT}

We do not know the origin of the Martian moons Phobos and Deimos. One set of hypotheses holds that they formed in the outer solar system or outer asteroid belt and were captured later by Mars [1][2], while others maintain that they formed in-situ near Mars, from a circumplanetary disk left over at the formation of Mars (e.g., [3][4]) or as a result of a giant impact [5].

These two broad classes of hypotheses predict different compositions (Table 1). If Phobos and Deimos were captured from the outer asteroid belt or outer solar system, their compositions are expected to resemble the most common asteroids; if they formed in-situ near Mars their compositions should be similar to Mars, i.e., ordinary chondrite, and, if formed by a giant impact, their compositions should consist of a mixture of Martian basalt depleted in volatile species such as water and potassium by impact heating, and similarly volatile depleted (but possibly carbon- rich) impactor material [6].

Some evidence for the capture scenario comes from remote-sensing observations of Phobos' and Deimos' surfaces, which suggest that they are similar to outer-belt asteroid material (e.g., [7][8]). However, the remote-sensing observations are ambiguous because the features that are seen can potentially be interpreted in multiple ways (e.g., [9]. This is partly due to space weathering in both the solar wind and Mars' magnetosphere, which has modified the spectra and washed out the

CubeSats and NanoSats for Remote Sensing II, edited by Thomas S. Pagano,

Charles D. Norton, Proc. of SPIE Vol. 10769, 107690I · (c) 2018 SPIE

CCC code: $0277-786 \mathrm{X} / 18 / \$ 18 \cdot$ doi: $10.1117 / 12.2322415$ 
strong absorption edges needed for compositional analysis. Overall, spectral evidence shows a low albedo, red-sloped near-infrared (NIR) spectrum similar to D-class asteroids, consistent with an origin in the outer asteroid belt or beyond. Alternatively, a silicate composition for Phobos has been suggested from Mars Express (MEX) mid-infrared (MIR) data, consistent with an in-situ formation (e.g., [9]).

Table 1. Possible origins and their Compositional Implications



The relationship between Phobos and Deimos has additional uncertainties: both may be pieces of a once-larger object, they may have resulted from separate captures, or they may have exchanged significant material. Only by obtaining a measurement of the large-scale compositional distribution of material in the Martian system can questions regarding the origins of Phobos and Deimos begin to be addressed. A robust and sensitive technique for obtaining elemental composition of surfaces in high vacuum environments is the collection and mass analysis of secondary ions ejected during ion and meteorite impacts. First proposed for space-based compositional analysis over thirty years ago (e.g., [10] [11]), this technique is commonly known in the laboratory as SIMS and can achieve excellent signal to noise ratios due to low ion backgrounds and high detection sensitivities achievable. Although ion-sensitive mass spectrometers have been flown on many missions previously, there has never been an instrument solely dedicated to measurement of ions sputtered from a small body surface by solar wind and magnetospheric plasmas.

PRISM would fill this gap by conducting SIMS measurements of both moons. MMX's direct measurement of the elemental composition of Phobos would allow the technique to be calibrated for the surface environment of the Martian moons, thus recovering the abundances of sufficient major elements to resolve the origin of Deimos and thus (with MMX), the Martian moon system.

SIMS measurements at Phobos and Deimos can resolve additional science questions. For instance, do these bodies harbor any potentially valuable resources for future exploration missions? Also, what compositional differences, if any, are observed in association with color differences on Phobos' surface? Currently it is not clear how spectral differences between the "red" unit, which exhibits a strong red slope at all near-IR wavelengths, and "blue" unit on Phobos' surface which is spectrally more neutral, correlate with compositional differences, if at all [6]. Like our Moon, the red unit contains $\mathrm{OH}$, though its origin as an intrinsic component of Phobos or a product of reaction with the solar wind remains unclear [8] [12]. The $\mathrm{OH}$ content of the blue unit appears to be less [8]. Questions that can be addressed by SIMS measurements thus include:

- How does the composition of Phobos' and Deimos' regolith compare to that of our own Moon and meteorite analogs?

- How much OH, as well as other volatiles, are on Phobos, and are they native or due to solar wind implantation?

- Is the red unit on Phobos dust that has migrated from Deimos [13]?

Using SIMS to directly sample the composition of Phobos and Deimos at different surface locations will help resolve these questions. 


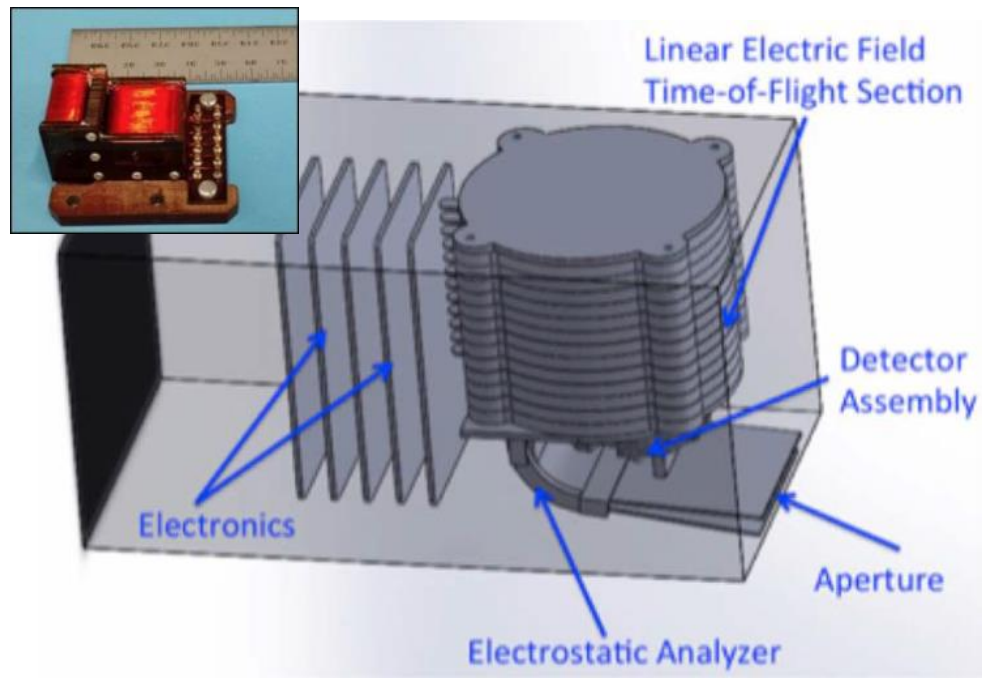

Figure 1 Proposed PRISM Instruments. (A) Ion Mass Spectrometer (IMS) solid model with (B) Magnetometer engineering model (insert).

\section{PROPOSED INSTRUMENTATION}

PRISM has two science instruments, an Ion Mass Spectrometer (IMS) and a Magnetometer (MAG) (Figure 1 a and b). The PRISM IMS is the main instrument and critical to achieving the science objectives. The PRISM Magnetometer is not a required instrument and could in principle be descoped. However, its data will make interpreting the IMS ion data significantly easier and increase the level of confidence in the interpretation of the IMS data.

The PRISM IMS is designed to achieve high time resolution in order 1) to provide spatial information about different sides of Phobos, 2) to capture environmental variability and 3) to allow investigators to select time periods when the motional electric field is guiding ions from Phobos to PRISM. We anticipate that we would have a general idea of the prevailing plasma flow in most regimes.

The magnetometer is included as part of the baseline PRISM instrument complement because of lessons learned from the LADEE experience [14]: analysis of the lunar pickup ions was greatly facilitated by having the magnetic field data from ARTEMIS magnetometer. These measurements enabled the reliable removal of the background from spacecraft- generated sources. Secondly, the high energy resolution of the PRISM IMS would allow the suppression of spacecraft-produced background even more effectively than the LADEE NMS. While LADEE measured an aggregate of all ions below about $25 \mathrm{eV}$ including thermal ions from very close to the instrument, PRISM would be be able to separate out the local ions based on energy [14]. Thus, the PRISM twofold approach enables unambiguous separation between local ions and ions originating at the Martian moons.

\section{SECONDARY ION MASS SPECTROMETRY}

The space environment continually liberates material from the regoliths of airless bodies throughout the solar system. Sputtering by the solar wind and energetic oxygen ions accelerated in Mars' magnetotail [15] [16] [17] [18], as well as more sporadic micrometeoroid impacts, create ions (as well as neutrals that may be subsequently ionized) in their hot impact plumes.

Laboratory work using lunar soils and analog materials has clearly shown that the composition of surfaces can be determined using a compact SIMS instrument to detect sputtered secondary ions [19] [20]. These studies showed that secondary ions are not sputtered in direct relation to the elemental concentration at the surface but depend on the elemental ionization potentials of the various species. Using relative secondary ion yields measured in sputtering experiments for known composition in the laboratory - such as a returned sample from the Martian moons - the observed secondary ion fluxes can be converted into relative abundances of the elements in the target material [21]. Additional measurements have shown that SIMS can detect the trace presence of $\mathrm{OH}$ in lunar samples (e.g., [22] [23] [24]. Using sputtered flux estimates obtained from experiments and Monte Carlo modeling of solar wind sputtering, Elphic and coworkers [19] and Schaible and coworkers [21] have demonstrated that the solar wind creates sufficient secondary ions for SIMS to provide successful composition measurements at airless bodies such as Phobos and Deimos. Additionally, these moons are exposed to energetic oxygen ions accelerated in Mars' magnetotail which can generate equivalent or larger sputtered fluxes than the solar wind [15] [16] [18].

Figure 2 shows secondary ion sputtering composition ratios for several compositional analogs to solar system bodies. The secondary ion yields were scaled to $\mathrm{Si}$ which has a similar abundance for each of the meteorite types considered. These 


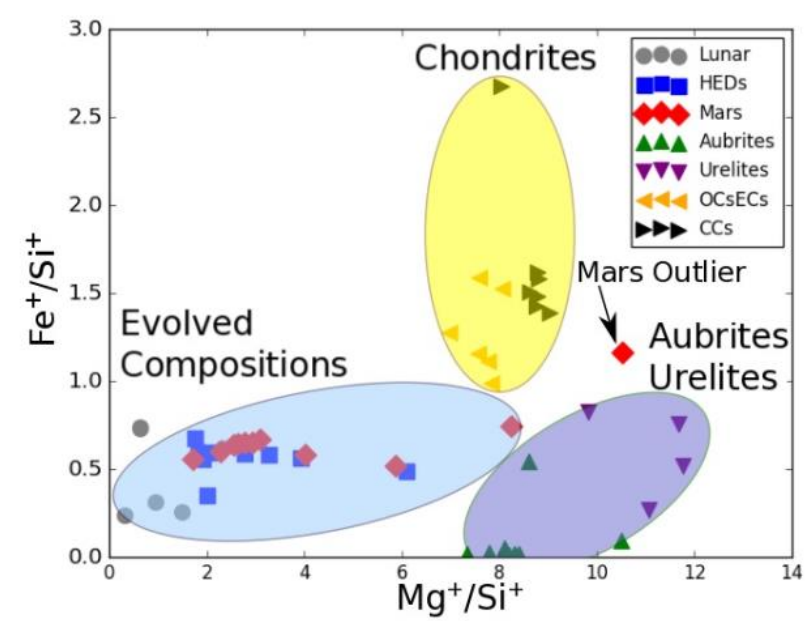

Figure 2 [21]: Sputtered secondary ion ratios for a representative suite of meteorite compositions. The ratios show a clear separation between evolved bodies such as the Moon, Mars, and the Howardite, Eucrite, Diogenite (HEDs) meteorite class and the primitive bodies such as ordinary and enstatite chondrites (OCsECs) and carbonaceous chondrite. Modified from Schaible et al. 2017. composition ratio plots show a separation between evolved compositions such as that of the surfaces of the Moon and Mars and the Howardite/Eucrite/Diogenite (HEDs) meteorite class, and primitive compositions such as ordinary and enstatite chondrites (OCsECs) and carbonaceous chondrites (CCs). In this particular plot, comparing more varied elements such as iron and magnesium, the primitive bodies (e.g., CCs, right facing triangles in Figure 2) and evolved bodies (e.g., Mars, red diamonds in Figure 2) are clearly distinguishable. The predicted secondary ion fluxes produced by solar wind and magnetotail oxygen sputtering are measurable using an ion mass spectrometer on a suitably formulated mission. With a thorough ground calibration program, compositional information obtained by PRISM will be sufficient by itself - even in the absence of calibration using MMX measurements - to resolve whether Phobos and Deimos are evolved or primitive bodies. Furthermore, SIMS observations can determine the abundance of $\mathrm{H}$ at Phobos, providing new insights into the $\mathrm{OH}$ and $\mathrm{H} 2 \mathrm{O}$ abundance on this moon.

The PRISM IMS fulfills the critical science need of detecting heavy ions sputtered from the surface of Phobos and Deimos with high sensitivity and resolving their species with better

than 1 amu mass resolution for masses up to at least 28. The IMS achieves this goal by utilizing an electrostatic gating technique, coupled with a linear-electric-field time-of-flight (TOF) section.

As shown in Figure 1, the PRISM IMS consists of an entrance aperture nominally oriented towards the surface of the target moon, an electrostatic analyzer section to select incident ions by energy per charge, an electrostatic ion gate and driver to produce well-defined start pulses of ions, a linear-electric-field TOF to focus ions of each species in time of flight, a microchannel plate detector to register each incident ion and produce a measurable charge pulse, and constant fraction discrimination and time-to-digital conversion circuitry to register the resulting time of flight spectrum.

The IMS electrostatic analyzer consists of two curved plates, with a programmable electric field between them, which selects incident ions by energy per charge. Figure 3 (top) shows the energy response of the analyzer, which has an analyzer constant (energy/voltage) of about 7.2 and a fractional energy resolution of about $12.5 \%(\Delta \mathrm{E} / \mathrm{E})$. The electrostatic analyzer, together with the entrance aperture and the exit aperture into the TOF, also defines the angular response of IMS. As shown in Figure 3, the PRISM IMS has an angular response of about $5^{\circ} \times 10^{\circ}$ Full-Width-Half-Maximum (FWHM), which will always allow a separation of particles coming from Phobos and, under some conditions, allow a separation of particles coming from the blue unit. The minor irregularities apparent in the polar angle response in the top panel of Figure $\mathbf{3}$ (middle) results from the presence of individual wires in the electrostatic gate assembly that is after the analyzer.

The PRISM IMS electrostatic gate utilizes a design of the Bradbury-Nielsen variety, consisting of an array of wires with equal and opposite voltages. This design has the advantage of a very low capacitance and very little leakage field at distances greater than the wire spacing, allowing rapid switching of the wire voltages to open (setting both voltages to zero) and close (setting voltages to $+/-10 \mathrm{~V}$ ) the gate and allowing a well-defined sharp packet of ions into the TOF. Significant development has been performed on the electrostatic gate at the University of Iowa, utilizing internal funding. This development includes prototyping the ion gate, mounting the prototype gates onto PCB with the driver circuitry, and simulating the ion trajectories through the gate.

The PRISM IMS utilizes the classic design for a time- of-flight section with a linear electric field, which establishes a harmonic-oscillator potential that ensures constant time-of-flight for species of a given mass per charge. The PRISM approach utilizes a stack of insulating spacers with electrodes between them of decreasing internal radius, establishing a pyramidal time-of-flight chamber with a potential very closely approximating the ideal harmonic form. Ions enter this 
TOF section from the exit of the analyzer, passing through the electrostatic gate. Simulations show perfect rejection to the walls of ions that pass through the gate when closed (Figure 4, red trajectories), but high throughput of ions when the gate is opened to define start pulses (Figure 4, green trajectories). Ions follow harmonic trajectories through the TOF section and are incident on the microchannel plate detector (labeled "Detector Assembly" in Figure 4), which utilizes secondary electron multiplication to produce detectable charge pulses.

Simulations show that this approach results in a time-offlight resolution $(\mathrm{t} / \Delta \mathrm{t})$ of greater than $\sim 100$, corresponding to a mass resolution $\mathrm{M} / \Delta \mathrm{M}$ greater than $\sim 50$. This is more than sufficient mass resolution to meet the PRISM science objectives. Simulations indicate that this design has a geometric factor of about $10^{-4} \mathrm{~cm}^{2} \mathrm{sr} \mathrm{eV} / \mathrm{eV}$.

The PRISM IMS electronics consist of the ion gate driver circuitry, high-voltage supplies for the electrostatic analyzer and TOF, a small microchannel plate detector to register ion counts and produce measurable charge pulses, a constant fraction discriminator and time-to-digital converter to accumulate time-of-flight spectra, limited digital functionality instantiated as firmware in an FPGA to control the ion gate and high voltages and packetize measured energy- TOF spectra, and low-voltage power conversion to produce regulated analog and digital voltages from unregulated bus voltage.

\section{MEASUREMENT METHODOLOGY}

Using its high-resolution time-of-flight (TOF) plasma composition analyzer, a miniaturized version of composition instruments that have flown for many years on a variety of spacecraft (e.g., [25]) the PRISM smallsat would observe species sputtered from Phobos' and Deimos' surfaces. The SIMS technique and ion mass spectrometers in general complement and expand quadrupole mass spectrometer measurements by collecting ions that are ejected from the
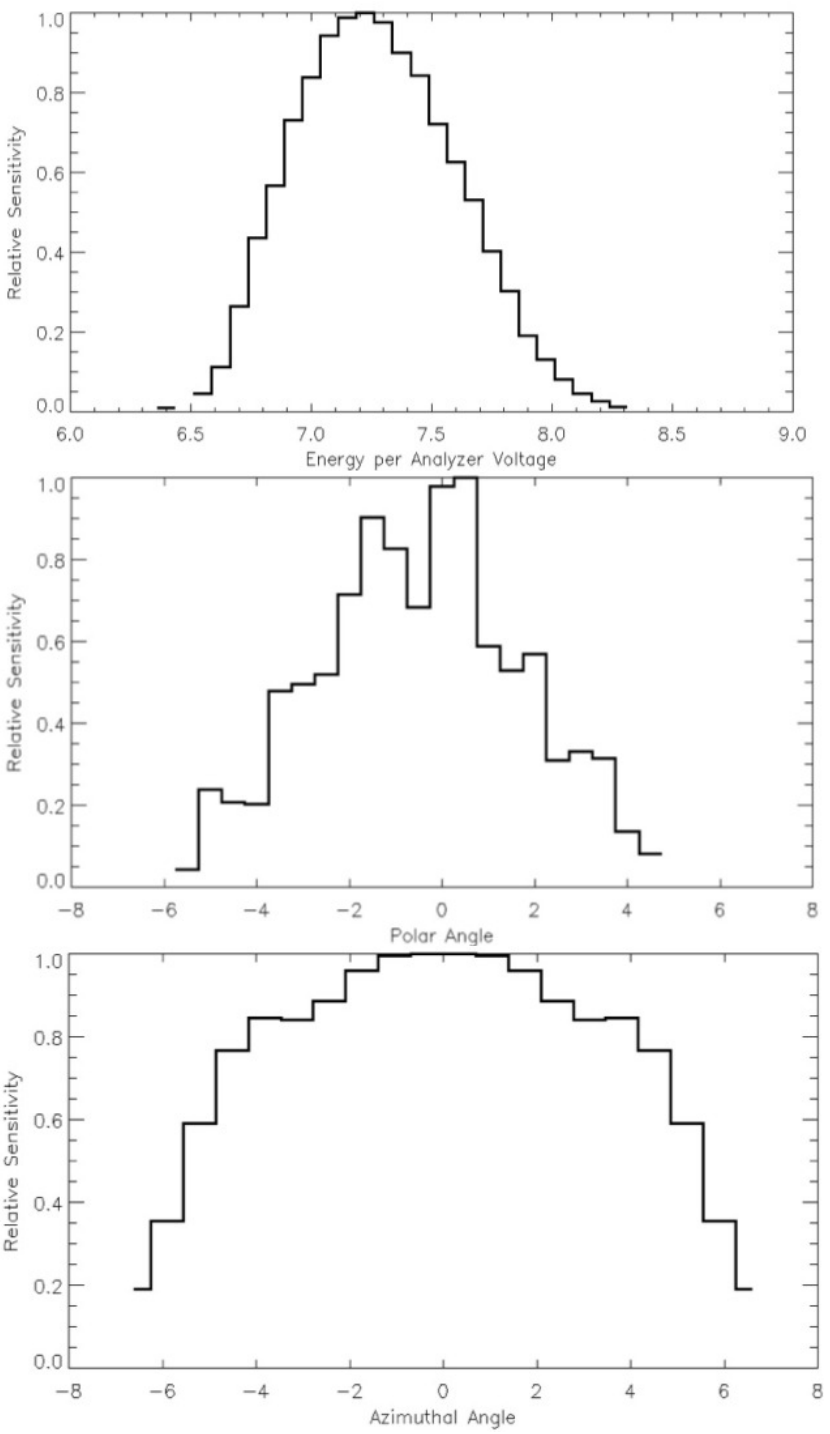

Figure 3: PRISM IMS simulated energy response (Top), polar angular (middle) and azimuthal (Bottom) angular response.

surface at energies of $\sim 5-50 \mathrm{eV}$. Due to low ion backgrounds from spacecraft and ambient sources and high instrumental sensitivities, measurements can be made at very low densities and pressures. Furthermore, because the TOF technique accepts all masses simultaneously, it does not require stepping through masses, a process that decreases the efficiency.

Pickup ions have already proven a powerful method to probe the composition and structure of the lunar surface and exosphere. Lunar pickup ions have been detected by many missions including AMPTE [26], Wind [27], Kaguya [28] [29], ARTEMIS [30] and LADEE [14]. Mars Express observations even show that the same solar wind proton backscattering that occurs at the Moon also occurs at Phobos [31]. These measurements establish that observable fluxes of ions generated by the interaction of the body with the space environment occur at the Moon and, even given the decrease in solar wind flux from Earth's orbit to the orbit of Mars, observable fluxes also exist at Phobos and Deimos. Although some metal ions such as $\mathrm{Ca}+$ and $\mathrm{Na}+$ have been observed, the general composition of the surface has not been obtained in previous exospheric ion measurements.

Phobos and Deimos, like the Moon, are airless bodies, and as such are exposed to energetic solar wind and oxygen ions which in turn eject secondary ions from the surface grains. Collection and analysis of these secondary ions can provide 


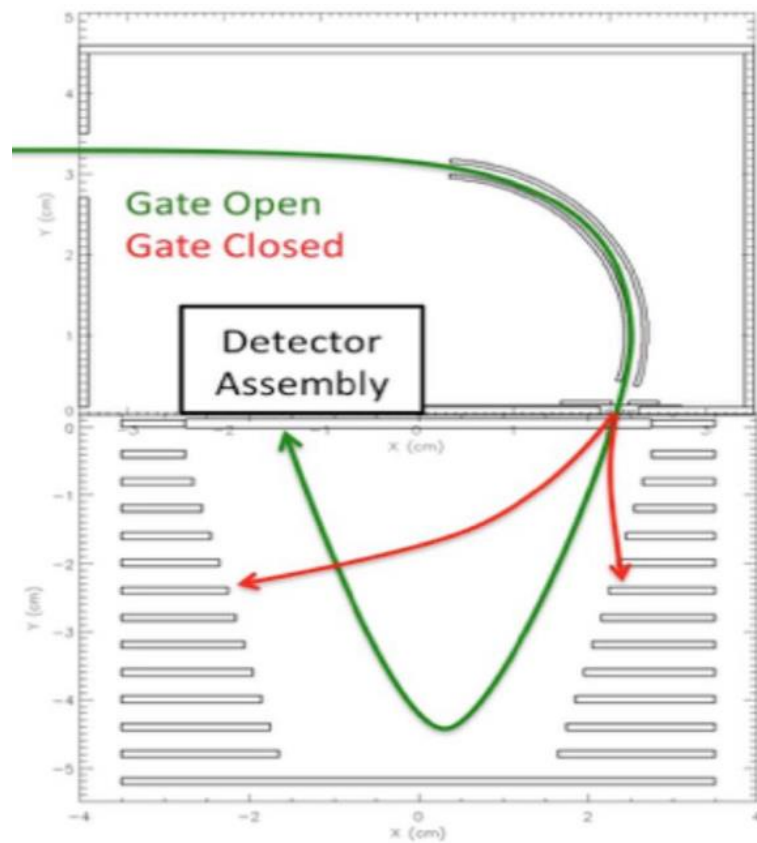

Figure 4 PRISM IMS linear electric field optics overview. bulk composition measurements from which specific geochemical inferences can be drawn (see Figure 1). Ion mass spectrometers do not rely on impact ionization of particles and are particularly sensitive to refractory elements which will be the major constituents of plausible Phobos and Deimos surface compositions. These species include $\mathrm{Fe}, \mathrm{Si}, \mathrm{Na}, \mathrm{Mg}, \mathrm{Al}$, and $\mathrm{Ca}$ [15] all of which have been shown to have relatively high secondary ion yields because of their relatively low ionization energy $(<9 \mathrm{eV})$ [19]. Either in complement to or independent of MMX results, examination of the relative abundances of major refractory elements ejected will provide important compositional measurements for the Martian moons. By performing laboratory calibration of measured abundances from representative materials such as meteorites, SIMS can determine the major elemental compositions of both Phobos and Deimos as a whole and investigate variations between distinct regions of Phobos, specifically the red and blue units (e.g., [32] [33]). If MMX is successful, calibration of both measurement sets to MMX measurements of Phobos' composition will reduce uncertainties in Deimos' composition due to possible differences in regolith texture and physical environment based on the laboratory measurements with compositional analogs described above.

Time-of-flight mass spectrometers have flown many times on

satellite missions and have high heritage (e.g., [34] [35]). There are two broad approaches to designing TOF (time- offlight) spectrometers, the thin foil approach (e.g., [25]) and the electronic gating approach (e.g., [36]). In both cases, the high mass resolution requirements of the science objectives $(M / \Delta M \geq 28$, to distinguish $\mathrm{Mg}$ (24) from $\mathrm{Si}(28)$ ) may require an isochronous time-of-flight unit that employs a linear electric field or, equivalently, parabolic potential (e.g., [25]. For both of these approaches, the conservative estimates suggest that the expected fluxes are large enough to achieve the science goals.

At an orbital distance of 2.76 Martian radii, Phobos is exposed to diverse plasma environments throughout its orbit around Mars. The primary sputtering sources are the solar wind and pick-up ions in the Martian magnetosphere. Because Phobos is phase-locked with Mars, the same hemisphere of Phobos faces Mars at all times such that the Mars-facing and antiMars faces of Phobos are exposed to different fluxes, energies, and compositions of the incident plasma. Phobos is "upstream" of Mars, exposing the anti-Mars hemisphere predominantly to $\mathrm{H}^{+}$and $\mathrm{He}^{2+}$ ions from the solar wind, while the majority of the bombardment of the Mars-facing hemisphere occurs in the magnetotail where the fluxes are dominated by $\mathrm{O}^{+}$and $\mathrm{O}^{2+}$ ions originating in the Martian atmosphere. Because rotation does not average out the exposure, it is important to take into account the different plasma environments at various locations on Phobos.

Total sputtering yields and generation of secondary ions produced by the solar wind and Martian oxygen ions may differ significantly. The solar wind is highly directional, coming from the sun, and has a high Mach number. In contrast, there are many different ion populations coming from Mars, and the oxygen energy can vary widely, as shown in Figure 5.

To best determine the sputtered fluxes due to the Martian environment from Phobos and Deimos, actual particle spectra from MAVEN were used. The Mars' environment is dynamic, resulting in no "representative" spectrum in the Martian tail (although the Mars' magnetosheath has some broadly reproducible features). So, a couple example spectra were chosen in the tail that contain relatively common features.

\section{MISSION CONCEPT}

At present, a CubeSat starting in Earth orbit (LEO) cannot get to Mars with its own propulsion. On the other hand, the likelihood of the PRISM CubeSat being manifested on a mission to Mars and being deployed in-orbit at Mars is low. So, our assumption in the trajectory analysis is that we are deployed outside of Earth's gravity well with a launch energy typical of missions heading to Mars. We refer to this as "The Mars 2020 Scenario" although this approach will work for 
any spacecraft headed for Mars changing the dates listed here by approximately 780 days (one Earth-Mars synodic period).

Even if PRISM piggybacks on a ride heading to Mars, it is likely that the host spacecraft (or upper stage - PRISM on the back side of the stage - or from an ESPA ring) will prefer to release any piggybacking CubeSats as early in the mission as possible. If PRISM were released closer to Mars arrival, PRISM would proceed with its Mars flyby which is needed to reduce the encounter velocity upon its return to Mars.

Figure 6 provides a graphical overview of the PRISM CONOPS. A bit over half a year following launch, there will be a close Mars flyby at $1815 \mathrm{~km}$ altitude, about

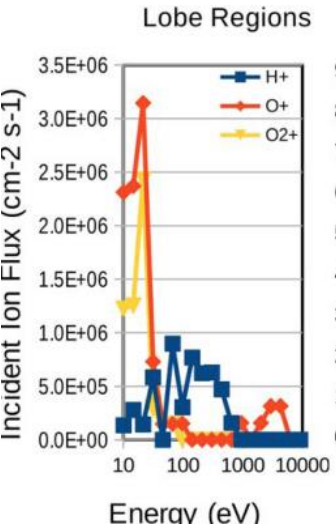
Plasma Sheet Magnetosheath half a Mars radius from the surface. This flyby will provide the opportunity to turn on the instruments, make sure that they are functional, and then get calibration data in the Mars system in preparation for Mars capture which will occur about three years later in February 2024 at a radial distance from Mars of 170 RM.

In addition, during the close flyby of Mars, PRISM may be able to perform some discovery science. For example, if the encounter could be arranged so that PRISM passes Mars on the night side, PRISM could observe cold ion outflow, composed primarily of $\mathrm{H}+, \mathrm{O}+$, and $\mathrm{O}_{2+}$ (with $\mathrm{CO}_{2}+$ also reported at lower levels), in the tail lobes. However, in theory other species such as $\mathrm{NO}+, \mathrm{C}+$, and $\mathrm{CO}+$ should be escaping: these are present in the Martian ionosphere, but have never been observed because of the required mass resolution. With PRISM's IMS, these species would be observable and PRISM observations during the flyby could confirm that they are actually escaping.

Following Mars capture in February 2024, there will be a one-year spiral-in phase for PRISM to rendezvous with Deimos at 6.9 RM. The distance to Phobos during the spiral-in phase will decrease approximately linearly with time allowing PRISM adequate time to characterize the Martian environment during this spiral-in phase. Following about three months at Deimos, PRISM will execute a one-year transfer orbit spiraling down to Phobos at about 2.8 RM and arrive in May 2026. After about three months in orbit about Phobos, the PRISM mission will end.

Note that the baseline schedule has PRISM arriving near Mars' perihelion, so that PRISM is relatively close to the sun and thus has more power available for thrust. When PRISM transfers from Deimos to Phobos, Mars is passing through aphelion, so that the available power and hence the available thrust drops considerably. This means that some of the Deimos to Phobos spiral is merely coasting in a circular orbit until Mars moves closer to the sun. This is why the transfer to Phobos takes about as long as the arrival to Deimos.

This effect could, in principle, be used to PRISM's advantage by shifting the schedule slightly. For example, if PRISM remains at Deimos for a few months longer until after Mars has passed through aphelion before spiraling to Phobos, PRISM would save spiral time and have more dry mass available.

The Mars Moons eXploration (MMX) mission will provide a sample return from the Martian moon Phobos in the 2020 s. However, MMX will only perform a flyby of Deimos without a sample return and provide insufficient time for nuclear spectroscopy at Deimos.

The composition of the returned samples in conjunction with laboratory SIMS measurements will provide a calibration for the PRISM IMS sputtering data. Because PRISM will co-orbit Deimos for many months, accumulating very good statistics on the sputtered products during this phase, the PRISM SIMS measurements, well-calibrated using the MMX Phobos sample return data, will determine the origin of Deimos and hence the origin of the Martian moons system.

Even without the benefit of MMX data, the elemental ratios measured by PRISM during its Deimos co-orbit will establish the origin of both Phobos and Deimos, namely distinguishing between the capture scenario and accretion/cataclysm. 


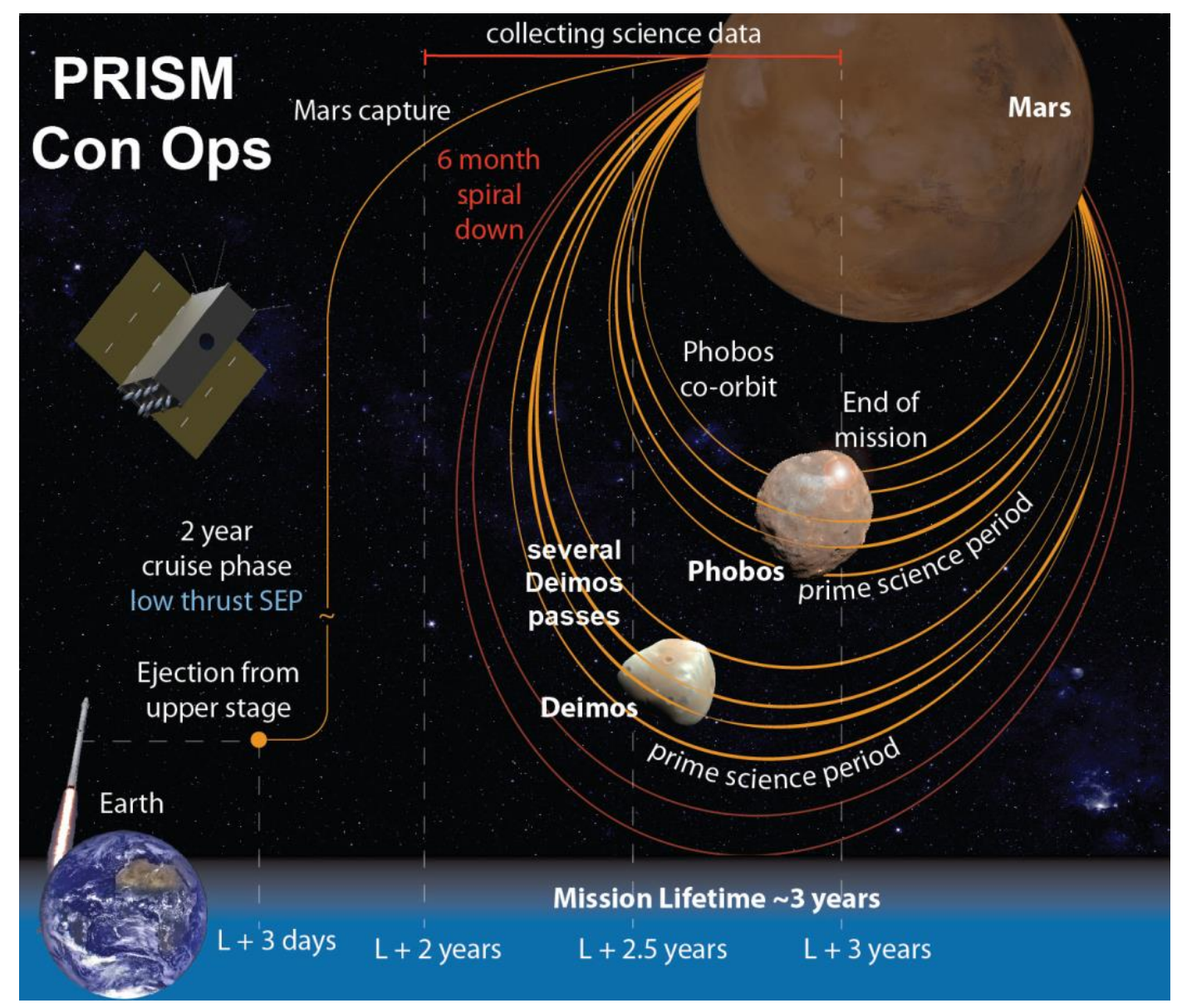

Figure 6: PRISM Operational Concept.

\section{ACKNOWLEDGMENT}

This material based on work supported by NASA under 16-PSDS316-0009 issued through the Planetary Science Deep Space SmallSat Studies Program. Portion of work at Jet Propulsion Laboratory, California Institute of Technology under contract with NASA. The information in this document is pre-decisional and is for information and discussion purposes only. (C) 2018. All rights reserved.

\section{REFERENCES}

[1] Burns, J.A. (1992), Contradictory clues as to the origin of the Martian moons, Mars, University of Arizona Press, Tucson, Kieffer et al. (eds), 1283-1301.

[2] Ivanov, A.V. (2004), Is the Kaidun meteorite a sample from Phobos?, Solar Sys. Res., 38, 2, 97- 107.

[3] Rosenblatt, Pascal, Sebastien Charnoz, Kevin M. Dunseath, Mariko Terao-Dunseath, Anthony Trinh, Ryuki Hyodo, Hidenori Genda, and Steven Toupin (2016), Accretion of Phobos and Deimos in an extended debris disc stirred by transient moons, Nat. Geosci., 9, 581-583, doi:10.1038/NGEO2742.

[4] Peale, S.J. and R.M. Canup (2015), The origin of the natural satellites, Treatise on Geophysics, Second Edition, 559604.

[5] Craddock, R.A. (2011), Are Phobos and Deimos the result of a giant impact?, Icarus, 211, 1150- 1161. 
[6] Murchie, S.L., P.C. Thomas, A.S. Rivkin, and N.L. Chabot (2015), Phobos and Deimos, in Asteroids IV (P. Michel et al., eds.), 17 pp., University of Arizona, Tucson.

[7] Pajola, M., et al. (2013), Phobos as a D-type captured asteroid, Spectral modeling from 0.25 to $4.0 \mu \mathrm{m}$, Astrophys. J., 777(2), 127.

[8] Fraeman, A.A., S.L. Murchie, R.E. Arvidson, R.N. Clark, R.V. Morris, A.S. Rivkin, and F. Vilas (2014), Spectral absorptions on Phobos and Deimos in the visible/near infrared wavelengths and their compositional constraints, Icarus, 229, 196-205, doi: 10.1016/j.icarus.2013.11.021.

[9] Giuranna, M., T.L. Roush, T Duxbury, R.C. Hogan, C. Carli, A. Geminale, and V. Formisano (2011), Compositional interpretation of PFS/MEX and TES/MGS thermal infrared spectra of Phobos, Planet. Space Sci., doi:10.1016/j.pss.2011.01.019.

[10] Managadze, G.G. and R.Z. Sagdeev (1988), Chemical composition of small bodies of the solar system determined from the effects of solar-wind interaction with their surfaces, Icarus, 73, 294-302.

[11] Johnson, R. and R. Baragiola (1991), Lunar surface-sputtering and secondary ion mass spectrometry, Geophys. Res. Lett., 18(11), 2169-2172, doi:10.1029/91GL02095.

[12] Farrell, W.M., D.M. Hurley, V.J. Esposito, J.L. McLain, and M.I. Zimmerman (2017), The statistical mechanics of solar wind hydroxylation at the Moon, within lunar magnetic anomalies, and at Phobos, J. Geophys. Res. - Planets, 122, 269-289, doi:10.1002/2016JE005168.

[13] Horanyi, Mihaly (2016), Dust Transport between the Martian Moons Phobos and Deimos, Phobos-Deimos Conference 2016, https://phobos-deimos.arc.nasa.gov/on-demand/.

[14] Halekas, J.S., M. Benna, P.R. Mahaffy, R.C. Elphic, A.R. Poppe, and G,T, Delory (2015), Detections of lunar exospheric ions by the LADEE neutral mass spectrometer, Geophys. Res. Lett., 42, doi:10.1002/2015GL064746.

[15] Cipriani, F., O. Witasse, F. Leblanc, R. Modolo, and R.E. Johnson (2011), A model of interaction of Phobos' surface with the Martian environment, Icarus, 212, 643-648.

[16] Dong, Y., X. Fang, D.A. Brian, J.P. McFadden, J.S. Halekas, J.E. Connerney, S.M. Curry, Y. Harada, J.G. Luhmann, and B.M. Jakosky (2015), Strong plume fluxes at Mars observed by MAVEN: An important planetary ion escape channel, Geophys. Res. Lett., 42, 8942-8950, doi:10.1002/2015GL065346.

[17] Harada, Y., J.S. Halekas, J.P. McFadden et al. (2015), Marsward and tailward ions in the near-Mars magnetotail: MAVEN observations, Geophys. Res. Lett, 42, doi:10.1002/2015GL065005.

[18] Poppe, A.R. and S.M. Curry (2014), Martian planetary heavy ion sputtering of Phobos, Geophys. Res. Lett., 41, doi:10.1002/2014GL061100.

[19] Elphic, R.C. et al. (1991), Lunar surface composition and solar wind-induced secondary ion mass spectrometry, Geophys. Res. Lett., 18, 216-218.

[20] Dukes, C.A. and R.A. Baragiola (2015), The lunar surface-exosphere connection: Measurement of secondary-ions from Apollo soils, Icarus, 255, 51-57.

[21] Schaible, M.J., C.A. Dukes, A.C. Hutcherson, R. Lee, M.R. Collier, and R.E. Johnson (2017), Solar wind sputtering rates of small bodies and ion mass spectrometry detection of secondary ions, J. Geophys. Res. - Planets, 122, doi: 10.1002/2017JE005359.

[22] McCubbin, F.M., A. Steele, E.H. Hauri, H. Nekvasil, S. Yamashita, and R.J. Hamley (2010a), Nominally hydrous magnetism on the moon, PNAS, 107, 25, 11223-11228.

[23] McCubbin, F.M., A. Steele, H. Nekvasil, A. Schnieders, T. Rose, M. Fries, P.K. Carpenter, and B.L. Jolliff (2010b), Detection of structurally bound hydroxyl in fluorapatita from Apollo mare basalt

[24] Boyce, J.W., Y. Liu, G.R. Rossman, Y. Guan, J.M. Eiler, E.M. Stolper, and L.A. Taylor (2010), Lunar apatite with terrestrial volatile abundances, Nature, Letters, 466, doi:10.1038/nature09274.

[25] Gloeckler, G. H. Balsiger, A. Burgi, et al. (1995), The solar wind and suprathermal ion composition investigation on the Wind spacecraft, Space Sci. Rev., 71, 79-124.

[26] Hilchenbach, M., D. Hovestadt, B. Klecker, and E. Mobius (1993), Observations of energetic lunar pick-up ions near Earth, Adv. Space Res., 13(10), 321-324.

[27] Mall, U. et al. (1998), Direct observation of lunar pick-up ions near the Moon, Geophys. Res. Lett., 25(20).

[28] Yokota, S. et al. (2009), First direct detection of ions originating from the Moon by MAP-PACE IMA onboard SELENE (KAGUYA), Geophys. Res. Lett., 36 (L11201).

[29] Tanaka, T. et al. (2009), First in situ observation of Moon-originating ions in Earth's magnetosphere by MAP-PACE on SELENE (KAGUYA), Geophys. Res. Lett., 36 (L22106). 
[30] Halekas, J.S., A.R. Poppe, G.T. Delory, M. Sarantos, W.M. Farrell, V. Angelopouslos, and J.P. McFadden (2012), Lunar pickup ions observed by ARTEMIS: Spatial and temporal distribution and constraints on species and source locations, J. Geophys. Res., 117 (E06006).

[31] Futaana, Y., S. Barabash, M. Holmstrom, A. Fedorov, H. Nilsson, R. Lundin, E. Dubinin, and M. Franz (2010), Backscattered solar wind protons by Phobos, J. Geophys. Res., 115, A10213, doi:10.1029/2010JA015486.

[32] Murchie, S.L., D.T. Britt, J.W. Head et al. (1991), Color heterogeneity of the surface of Phobos - Relationships to geologic features and comparison to meteorite analogs, J. Geophys. Res., 96, B4, 5925-5945, doi:10.1029/90JB02354.

[33] Murchie, S. and S. Erard (1996), Spectral properties and heterogeneity of Phobos from measurements by Phobos 2, Icarus, 123, 63-86.

[34] Gilbert, J.A., R.A. Lundgren, M.H. Panning, S. Rogacki, and T.H. Zurbuchen (2010), An optimized three-dimensional linear-electric-field time-of-flight analyzer, Rev. Sci. Inst., 81, 053302.

[35] Hilchenbach, M. (2002), Space-borne mass spectrometer instrumentation, Int. J. Mass Spec., 215, 113-129.

[36] Keller, John. W., Dennis J. Chornay, Floyd H. Hunsaker, Keith W. Ogilvie, and Michael A. Coplan (1999), Gated time-of-flight plasma composition analyzer for space physics research, Rev. Sci. Inst., 70, 7, 3167-3172. 